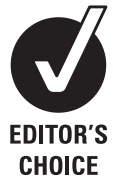

${ }^{1}$ Norfolk and Norwich University Hospital NHS Trust, Norwich, Norfolk, UK

${ }^{2}$ NHS Blood and Transplant, Addenbrookes Hospital, Cambridge, UK

\section{Correspondence to} Dr Julian Garside, Emergency Medicine Registrar, Norfolk and Norwich University Hospital NHS Trust, 48 Pightle Way, Lyng, Norwich, Norfolk NR9 5RL, UK;

julian.garside@nnuh.nhs.uk

Accepted 10 February 2011 Published Online First 17 March 2011

\title{
Utilisation of an embedded specialist nurse and collaborative care pathway increases potential organ donor referrals in the emergency department
}

\author{
Julian Garside, ${ }^{1}$ Marie Garside, ${ }^{2}$ Simon Fletcher, ${ }^{1}$ Bruce Finlayson ${ }^{1}$
}

\section{ABSTRACT}

Objective To review the impact of an embedded specialist nurse in organ donation (SN:OD) and the utilisation of a collaborative care pathway on potential solid organ donor referrals in an emergency department (ED) over a 24-month period.

Methods A retrospective cohort study of adult patient deaths within the department, $(n=311)$ during a 24-month period. Referral rates to the organ donation team (ODT) were compared before and after the introduction of a SN:OD and collaborative care pathway. Results Referrals to the ODT significantly increased from three of 151 eligible patients (2\%) to 26 of 160 patients (16\%; $\chi^{2}$ test $\left.p<0.0001\right)$ following the introduction of a SN:OD into the trust. The number of patients proceeding to organ donation increased from none to two (Fisher's exact test $p=1.0$ ).

Conclusions The presence of an embedded SN:OD in the ED and the adoption of a collaborative care pathway to establish clinical triggers for referral to the ODT have significantly increased the rate of referral of adult potential organ donors to organ donation services.

There are currently more than 8000 individuals awaiting organ transplantation in the UK. ${ }^{1}$

Historically, the organ donation process has been synonymous with intensive care units.

Advances in neurosurgical techniques, improved intensive care patient outcomes, and extensive road safety legislation has reduced the total number of brainstem dead donors and contributed to a significant disparity between the availability of donated organs and the increasing number of patients requiring transplantation. ${ }^{23}$

Each day three of these people will die because the organ they need is not available (figure 1). ${ }^{1}$ These deaths are potentially preventable.

At present only $28 \%$ of the UK population have registered their wish to donate organs by joining the NHS Organ Donor Register, despite $90 \%$ of the UK population supporting the concept of organ donation. $^{4}$

In order to address this situation the Department of Health commissioned the Organ Donation Taskforce in 2006 to reappraise the UK model for organ donation. Their recommendations were published in $2008 .^{1}$

Within this report, the introduction of embedded specialist nurses in organ donation (SN:OD) was recommended in every acute trust, and the potential role of the emergency department (ED) in the identification and referral of patients suitable for organ donation was highlighted. ${ }^{1}$
We undertook a review of deaths in one $\mathrm{ED}$ between March 2008 and August 2010 to assess the effect on organ donor referral rates of an in-house $\mathrm{SN}: \mathrm{OD}$ and the utilisation of a collaborative care pathway.

\section{ORGANISATION}

The ED of the Norfolk and Norwich University Hospital NHS Foundation Trust sees approximately 85000 new patients per year, with a catchment population of 650000

A SN:OD has been embedded in the Norfolk and Norwich University Hospital NHS Foundation Trust since March 2009, funded by NHS Blood and Transplant as part of the Eastern Organ Donation Services Team.

The Eastern Organ Donation Services Team consists of $10 \mathrm{SN}$ :OD who provide $24 \mathrm{~h}$ cover to 19 $\mathrm{ED}$ and 23 intensive care units.

Twenty-four hour provision of this service is provided via an on-call specialist nurse covering the region, contacted through a single mobile phone number.

During the 12-month period between the appointment of our $\mathrm{SN}: \mathrm{OD}$ and the endpoint of our study in February 2010, a significant proportion of time has been spent in service development at trust management level as well as tutoring newly appointed SN:OD and in the continuous education of clinical staff and trust management in the importance of a successful organ donation programme within the trust. Now this service has been developed, the aim is to sustain awareness of the importance of organ donation through continued education, SN:OD presence in clinical areas, and the use of local and national media to improve public awareness.

The SN:OD role has been defined as 'to make donation a usual rather than an unusual event and an expected part of end-of-life care' and ensure that patients' end-of-life wishes are identified and acted upon as set out in the Human Tissue Act, $2006 .{ }^{5}$

This role of the SN:OD involves close liaison with intensive care and ED staff at all levels, ensuring a multidisciplinary collaborative approach to the early identification and management of potential organ donors. Early prolonged contact with donor families, an extensive staff education programme, and increasing public awareness of organ donation issues has been shown to increase organ donation rates. ${ }^{6}$

\section{STUDY POPULATION AND METHODS}

A retrospective review of all patient deaths within the ED of the Norfolk and Norwich University 


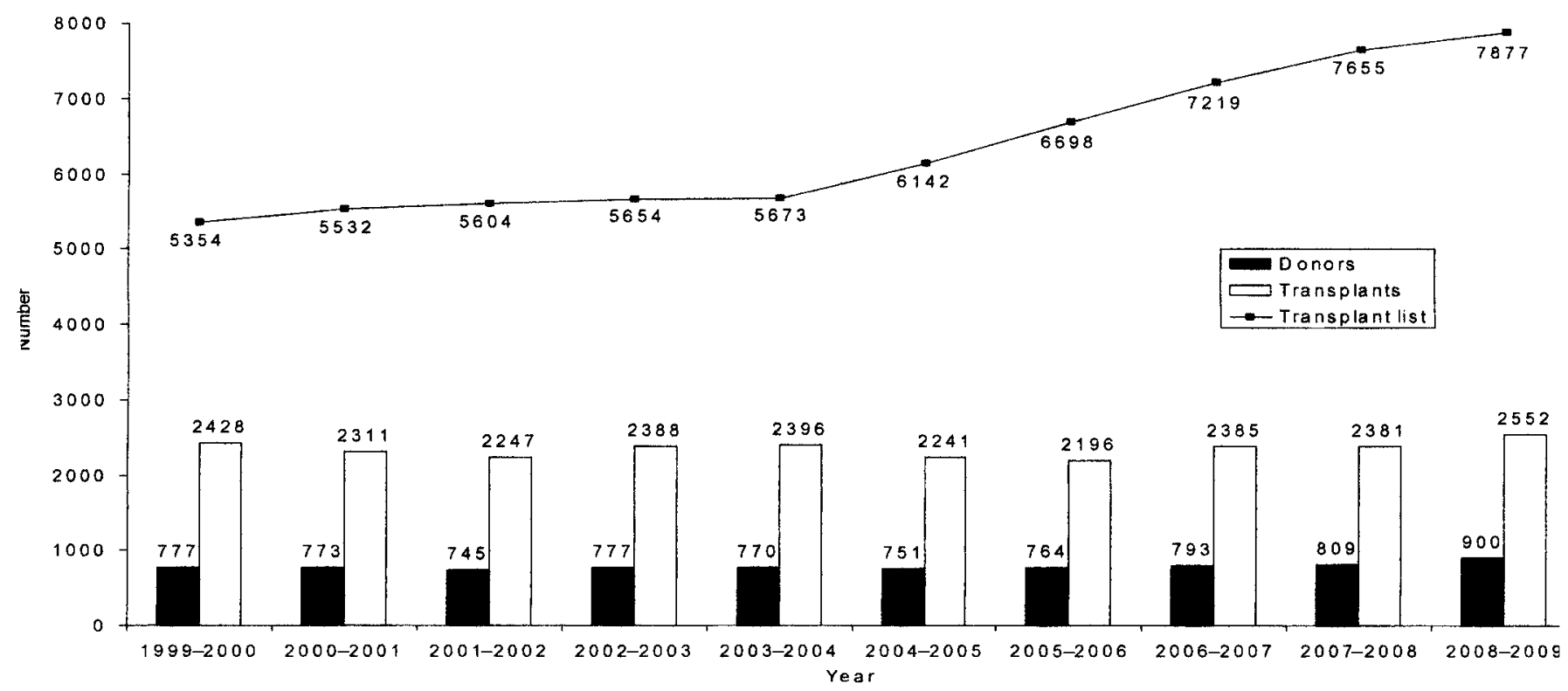

Figure 1 The transplant waiting list.

Hospital between 1 March 2008 and 28 February 2010 was undertaken through the examination of ED patient records.

Consecutive ED patient attendances, deaths, organ donation team (ODT) referrals and numbers of patients proceeding to solid organ donation were reviewed for the 12 -month period before the appointment of our SN:OD: March 2008 to February 2009.

These data were then compared with the 12-month period March 2009 to February 2010 after the appointment of the SN:OD.

Analysis of these two cohorts of patients was then undertaken to assess whether a statistically significant difference existed between the rates of referral for organ donation.

A collaborative care pathway, agreed by both $\mathrm{ED}$ and intensive care clinicians, was also introduced to identify clinical triggers and facilitate the referral of potential organ donors to the ODT via the embedded SN:OD (or duty SN:OD out of hours; figure 2).

It was agreed that any adult patient presenting to the ED with an unsurviveable condition, in which further medical treatment was deemed futile and when the decision to extubate terminally had been made (after consultation with senior ED, intensive care and neurosurgical clinicians) would be referred to the embedded SN:OD.

The SN:OD would then, after consulting the UK Organ Donor Register, assess the patient's suitability to become an organ donor and discuss the case with the duty transplant surgeons.

When patients were deemed medically unsuitable, the subject of organ donation was not raised with the family, unless they themselves requested organ donation.

If medical suitability was established, the SN:OD would work with clinical staff to counsel the family and obtain informed consent for organ donation to proceed.

Should the family decline consent for organ donation, the $\mathrm{SN}$ :OD would continue to provide valuable support to them throughout the bereavement process.

\section{RESULTS}

During the study period 1 March 2008 to 28 February 2010 there were 156401 patient attendances to the Norfolk and Norwich University Hospital ED, with 311 recorded deaths in the department $(0.20 \%$ death rate; table 1$)$.
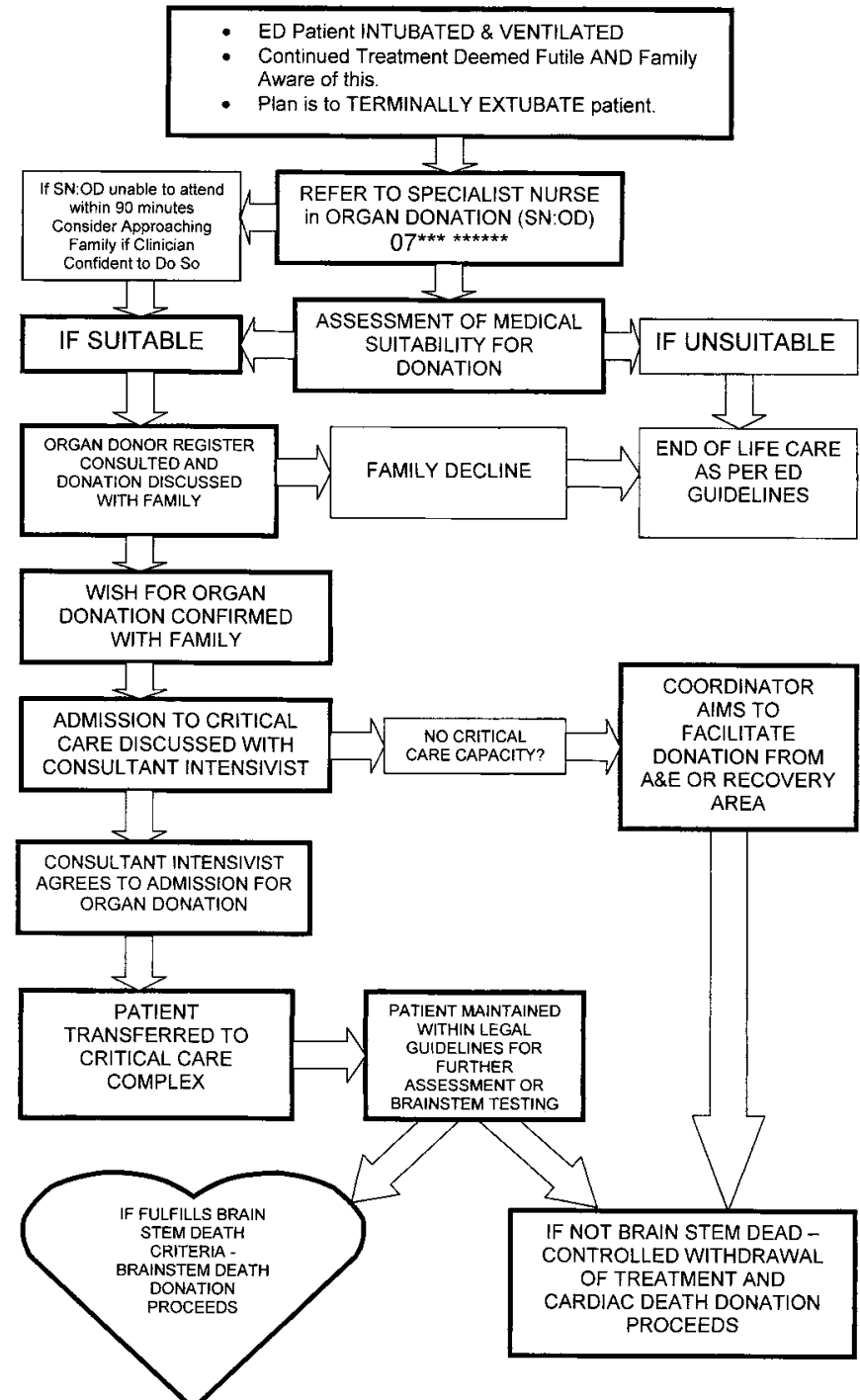

Figure 2 Collaborative care pathway. ED, emergency department; $\mathrm{SN}: 0 \mathrm{D}$, specialist nurse in organ donation. 
Table 1 ED demographic data, March 2008-February 2010

\begin{tabular}{llll}
\hline Time period & Mar-08-Feb-09 & Mar-09-Feb-10 & Total \\
\hline Total ED attendances & 75310 & 81091 & 156401 \\
Male (\%) & $40085(53.2 \%)$ & $42534(54.2 \%)$ & $82619(53.7 \%)$ \\
Female (\%) & $35225(46.8)$ & $38557(45.8 \%)$ & $73782(46.3 \%)$ \\
Total ED deaths & 151 & 160 & 311 \\
ED deaths (\%) & $0.20 \%$ & $0.20 \%$ & $0.20 \%$ \\
Male ED deaths & 83 & 91 & 174 \\
Male age range, years & $17-94$ & $21-98$ & 137 \\
Mean male age, years (95\% Cl) & $69(65$ to 73$)$ & $68(64$ to 71$)$ & 17.6 \\
Male SD & 19.7 & 69 & $34-99$ \\
Female ED deaths & 68 & $80(77$ to 84$)$ & 13 \\
Female age range, years & $29-99$ & 26 & 29 \\
Mean age, years (95\% Cl) & $78(75$ to 81$)$ & 2 & \\
Female SD & 12.5 & 3 & \\
Referrals to ODT & 0 & & \\
Patients proceeding to organ donation & 3 & & \\
\hline
\end{tabular}

ED, emergency department; ODT, organ donation team.

Of the 311 total ED deaths, 151 (48.5\%) were recorded during the 12-month period March 2008 to February 2009 with three referrals to the ODT being made and no (zero) patients proceeding to solid organ donation.

During the 12-month period March 2009 to February 2010, $160(51.5 \%$ of total) deaths were recorded, with 26 referrals to the ODT and two patients proceeding to organ donation.

Comparison of patient referral numbers from the ED and intensive therapy unit (ITU) over the same time period studied show that there was a global increase in patient referrals from both departments once the SN:OD was in post from March 2009 (figure 3, table 2).

\section{Analysis}

Comparison of the two cohorts of ED data was undertaken using a $\chi^{2}$ test to assess the statistical significance of the change in ODT referrals following introduction of an embedded $\mathrm{SN}$ :OD (table 3).

Analysis of the numbers of referred patients actually proceeding to organ donation was undertaken using Fisher's exact test in view of the relatively small sample sizes (table 4).

Statistical analysis of the data from the two cohorts studied indicates that the presence of an embedded SN:OD during the end-of-life process significantly increased the numbers of potential organ donors identified in the ED from three to $26(p<0.0001)$.

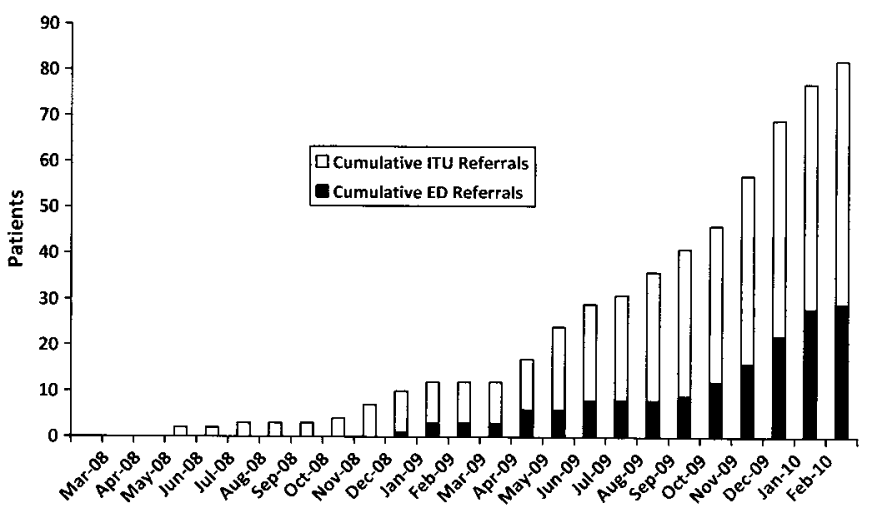

Figure 3 Cumulative emergency department and intensive therapy unit referrals, March 2008-February 2010. ED, emergency department; ITU, intensive therapy unit.
The increase in the number of patients actually proceeding to organ donation from zero to two was not statistically significant $(p=1.0)$. However, the small sample size suggests that follow-up analysis from several centres would be useful.

\section{DISCUSSION}

The Organ Donation Taskforce recommended that potential organ donors should be identified in the ED and embedded specialist nurses employed to facilitate organ donation from these donors. ${ }^{1}$

The process of organ donation has traditionally been seen as the remit of the critical care complex and neurosurgical intensive care unit as the majority of organs were donated by patients deemed brainstem dead on formal neurological testing (heart beating donors now classified as donor after brain death; DBD). However, the use of kidneys from non-heart-beating donors (now classified as donor after cardiac death; DCD) has increased from 3\% in 2000 to 34\% by September 2010. ${ }^{7}$

Moreover, recent research suggests that recipient outcome after transplantation of kidneys from DCD is 'good' compared with kidneys donated by $\mathrm{DBD}$, although further studies are indicated. $^{89}$

To date, no formal brainstem testing of potential donors is undertaken in our ED, mainly due to the fact that many intubated ED patients have been sedated to facilitate intubation in the prehospital setting. Logistical factors, such as the need for the presence of two relevantly registered experienced clinicians to confirm brainstem death also has to be considered in DBD donation. $^{10}$

The introduction of formal brainstem testing in the ED would enable DBD donation to proceed but also necessitate further education of $\mathrm{ED}$ staff in the physiological optimisation and maintenance of the $\mathrm{DBD}$, a process previously undertaken by experienced ITU staff.

This redeployment of ED staff could potentially increase the workload in an already busy department.

The introduction of a pathway enabling formal brainstem testing to occur in the ED would allow the DBD donation process to occur in ED rather than ITU and could improve the number of patients proceeding to solid organ donation.

However, these perceived obstacles to DBD donation from the $\mathrm{ED}$ are not insurmountable, particularly when the education and supervision of clinical staff can be undertaken by an embedded $\mathrm{SN}: \mathrm{OD}$ experienced in the care of the DBD. 
Table $3 \chi^{2}$ test-ED referrals to the organ donation team

\begin{tabular}{llll}
\hline & Referred & Not Referred & Total \\
\hline Mar-08-Feb-09 cohort & 3 & 148 & 151 \\
Mar-09-Feb-10 cohort & 26 & 134 & 160 \\
Total & 29 & 282 & 311 \\
p Value & $<0.0001$ & & \\
\hline
\end{tabular}

$\chi^{2}=19$ degrees of freedom $=1$.

ED, emergency department.

Increased awareness of the importance of organ donation in our ED has enabled the early identification of potential donors and their transfer to the critical care complex in order to undergo formal brainstem testing.

The presence of an embedded $\mathrm{SN}: \mathrm{OD}$ has been shown to increase the conversion of potential organ donors to actual organ donors. ${ }^{6}$ Early prolonged contact with donor families allows them to form a relationship with the specialist nurse and has been shown to increase the donor families' understanding of the donation process, also increasing consent rates. ${ }^{11}$

Informal feedback from donor families has also strongly suggested that prolonged contact with the SN:OD has helped them tolerate their grief and to feel better supported during the bereavement process.

Our data demonstrate that the presence of an embedded $\mathrm{SN}$ :OD has significantly increased the referral rate of potential organ donors both in our ED and from the intensive care unit, although the actual number of ED patients proceeding to solid organ donation did not significantly increase during the study period.

\section{Limitations of the study}

The non-significant increase in patients proceeding to solid organ donation is most likely due to two main factors: the relatively small sample size from a single trust; and the relatively elderly population in the catchment area of our ED, whose organs are more likely to be deemed medically unsuitable for donation by the transplant surgeons.

During the time period of our study, particularly before the appointment of the embedded SN:OD, no formal audit of potential organ donors was undertaken by NHS blood and transplant or individual donor transplant coordinators (the precursors to the current $\mathrm{SN}: \mathrm{OD}$ ), and therefore no formal data were available from our trust to compare 'missed' potential donor numbers before and after appointment of the SN:OD.

As a result of the lack of formal data regarding potential donors, it was difficult to ascertain the exact proportion of 'missed potential donors' during the 24-month study period.

In order to address this, a review of ED patient cards covering the 24-month study period was undertaken by the main author and current $\mathrm{SN}: \mathrm{OD}$

Before the appointment of our $\mathrm{SN}: \mathrm{OD}$, it was common practice for withdrawal of treatment to be initiated in the ED (ie, terminal extubation following review by the duty intensivist).

The extubated patient would then be moved to an appropriate ED area or ward where end-of-life care would be given.

Table 4 Fisher's exact test-ED referrals proceeding to organ donation

\begin{tabular}{llcc}
\hline Data analysed & Donation & No donation & Total \\
\hline Mar-08-Feb-09 cohort & 0 & 3 & 3 \\
Mar-09-Feb-10 cohort & 2 & 24 & 26 \\
Total & 2 & 27 & 29 \\
p Value & 1.0 & & \\
\hline
\end{tabular}

ED, emergency department. 


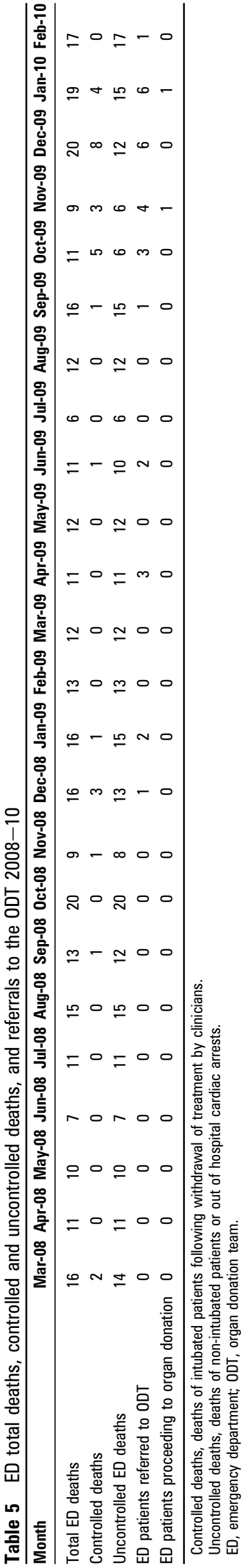

This led to potential DCD being extubated and moved out of the ED before any $\mathrm{SN}$ :OD referral could be made to assess their suitability for organ donation.

This is confirmed in table 5, in which three of the eight controlled ED deaths were referred to the ODT during the 12period before the appointment of the $\mathrm{SN}: \mathrm{OD}$.

Examination of the 12-month period after the introduction of the SN:OD and collaborative care pathway confirms both an increase in recorded controlled ED deaths (22) and ODT referrals from the ED (26).

The introduction of the NHS Blood and Transplant Potential Donor Audit, undertaken by all embedded SN:OD since April 2010, will enable a more accurate assessment of potential organ donors in the ED and the donor conversion rate from our trust.

This suggests that a prospective multicentre study using data from the potential donor audit may help to clarify whether our demonstrated increase in referrals for solid organ donation is replicated in other trusts where an embedded SN:OD is employed.

\section{CONCLUSION}

In 2009 there were 959 patients proceeding to solid organ donation in the UK. ${ }^{7}$

If each of the 211 UK ED could identify one additional donor each year, then this would increase donor numbers by $22 \%$ and, with an average of three transplantable organs per donor being retrieved, transform the lives of over 600 patients currently waiting for an organ transplant. The presence of a dedicated SN: $\mathrm{OD}$ in our trust has increased potential organ donor referral rates from our ED, raised staff awareness of organ donation and enhanced the bereavement care that donor families receive.

Acknowledgements The authors would like to thank Patrick Musonda, honorary lecturer and medical statistician, from the School of Medicine, Health Policy \& Practice, University of East Anglia, Norwich NR4 7TJ, UK.

Competing interests None.

Provenance and peer review Not commissioned; externally peer reviewed.

\section{REFERENCES}

1. Department of Health. Organs for transplant: a report from the Organ Donation Taskforce. London: Department of Health, 2007.

2. WHO European Health for All Database. Mortality trends for motor vehicle traffic accidents and cerebrovascular disease. http://www.euro.who.int/hfadb laccessed 24 Jan 2011).

3. Clayton TJ, Nelson RJ, Manara AR. Reduction in mortality from severe head injury following introduction of a protocol for intensive care management. $\mathrm{Br} J$ Anaesth 2004;93:761-7.

4. UK Transplant. How do we get more to register? Bulletin 2003;47:10-12.

5. Department of Health. Human Tissue Act, 2004. London: Department of Health, 2004. http://www.legislation.gov.uk/ukpga/2004/30/contents/enacted (accessed 8 Mar 2011)

6. Shafer TJ, Davis KD, Holtzman SM, et al. In-house organ procurement organization staff located in level 1 trauma centres increase conversion of potential donors to actual donors. Transplantation 2003; 75:1330-5.

7. National Health Service Blood and Transplant. Transplant activity in the UK, 2009-2010. http://www.organdonation.nhs.uk/ukt/statistics/latest_statistics/ monthly.jsp (accessed 24 Jan 2011).

8. Summers D, Johnson R, Allen J, et al. Analysis of factors that affect outcome after transplantation of kidneys donated after cardiac death in the UK: a cohort study. Lancet 2010;376:1303-11.

9. Dahmane D, Audard V, Heisse C, et al. Retrospective follow-up of transplantation of kidneys from 'marginal' donors. Kidney Int 2006;69:546-52.

10. Academy of Medical Royal Colleges. A code of practice for the diagnosis and confirmation of death. 2008. http://www.aomrc.org.uk/publications/reportsguidance/doc_download/42-a-code-of-practice-for-the-diagnosis-and-confirmationof-death.html (accessed 8 Mar 2011).

11. Siminoff LA, Gordon N, Hewlett J, et al. Factors influencing families' consent for donation of solid organs for transplantation. JAMA 2001;286:71-7. 